\title{
Resolución de localización espacial háptica mediante estimulación eléctrica en la yema de los dedos
}

\author{
Resolution of spatial localization haptic \\ by electrical stimulation in the fingertips
}

David Escobar Valencia ${ }^{1 *} \quad$ José María Vicente Samper ${ }^{2}$

José María Sabater Navarro ${ }^{2} \quad$ Oscar Andrés Vivas Albán $^{1}$

Recibido 27 de diciembre de 2018, aceptado 17 de diciembre de 2019

Received: December 27, 2018 Accepted: December 17, 2019

\begin{abstract}
RESUMEN
La estimulación eléctrica ha demostrado su utilidad como actuadores en el desarrollo de dispositivos hápticos, ya que presenta la posibilidad de variar parámetros como frecuencia e intensidad de corriente en diversas regiones del cuerpo humano, produciendo estímulos diferentes en los distintos órganos mecanorreceptores. Cuando se utiliza en la yema de los dedos permite la distribución de las señales a electrodos enmarcados en un arreglo tipo matriz, con el fin de producir distintas sensaciones en el usuario. Este trabajo describe, por medio de un prototipo basado en estimulación eléctrica, la resolución a efectos de localización espacial háptica. Se presentan los resultados de una primera experimentación con usuarios sanos.
\end{abstract}

Palabras clave: Estimulación eléctrica, localización háptica, dispositivo háptico, matriz de electrodos.

\section{ABSTRACT}

The electrical stimulation has demonstrated its usefulness as actuators in the development of haptic devices, since it presents the possibility of varying parameters such as frequency and current intensity in different regions of the human body, producing different stimuli in the different mechanoreceptor organs. When used on the fingertips, it allows the distribution of the signals to electrodes framed in a matrix type arrangement, in order to produce different sensations in the user. This work describes, by means of a prototype based on electrical stimulation, the resolution for the purpose of haptic spatial location. The results of a first experimentation with healthy users are presented.

Keywords: Electrical Stimulation, haptic location, haptic device, array of electrodes.

1 Universidad del Cauca. Departamento de Electrónica, Instrumentación y Control. Popayán, Colombia. E-mail: davidescobar@unicauca.edu.co; avivas@unicauca.edu.co

2 Universidad Miguel Hernández. Departamento de Ingeniería de Sistemas y Automática. Elche, España. E-mail: jose.vicentes@umh.es; j.sabater@goumh.umh.es

* Autor de Correspondencia: davidescobar@unicauca.edu.co 


\section{INTRODUCCIÓN}

El término háptica proviene del griego haptohapthestai, contextualizado como tocar. Por ende, suele relacionarse para describir todo aquello basado con el sentido del tacto, haciendo referencia de igual manera a aquella ciencia que estudia las sensaciones y percepciones producidas por la interacción del ser humano frente al entorno en el cual se encuentra [1-2].

Generalmente el sentido háptico se ve enmarcado por intercambios de tipo estáticos o dinámicos que proporcionan la captación de características físicas, estableciendo un papel de evaluación frente a algunos atributos del medio, debido a un proceso de trasmisión y recepción de información por parte de los usuarios [3-4]. Esto puede ser llevado a cabo por la yema de los dedos, ya que en la piel se encuentran múltiples terminaciones nerviosas. Desde el punto de vista fisiológico dichas respuestas se producen gracias a los mecanorreceptores cutáneos, siendo estos los corpúsculos de Meissner, los discos de Merkel, los corpúsculos de Pacini y finalmente las terminaciones de Ruffini, encargados de detectar los diferentes cambios que se producen en una superficie, respondiendo ante ellas a través de un impulso nervioso [5-6].

Para llevar a cabo estados de estimulación de esta región del cuerpo humano, las diferentes investigaciones reportadas en la literatura han prestado considerable atención a desarrollos de dispositivos táctiles empleando diversos actuadores, con el propósito de generar algún tipo de señal para producir una respuesta enmarcada en la sensación, incluyendo de igual manera atributos psicofísicos dentro de los experimentos realizados $[7,8,9]$.

No obstante criterios como la localización, la resolución espacial en la percepción de los estímulos, así como la calidad de la sensación han recibido poca atención [10-11]. De igual manera pocos estudios se han dedicado a determinar la distribución de la población en la interpretación de esta información por medio de subgrupos separados por sexo.

En este trabajo se presenta un sistema de estimulación eléctrica evaluado con tres experimentos en los cuales los participantes realizan tareas de localización espacial táctil por medio de secuencias eléctricas aplicadas en el dedo índice de su mano dominante, para posteriormente indicar y expresar de forma verbal el punto de localización del estímulo presentado.

Este documento se encuentra estructurado de la siguiente manera; la sección 2 presenta el estado del arte enmarcado en trabajos relacionados con la producción de sensaciones por medio de diversos actuadores sobre la yema de los dedos. La sección 3 describe el dispositivo implementado para el desarrollo de este trabajo. La sección 4 presenta un protocolo psicofísico aplicado en un grupo de participantes, con el objetivo de determinar la localización táctil por medio de estimulación eléctrica a través de tres experimentos planteados. Finalmente, la sección 5 presenta conclusiones y trabajos futuros.

\section{SISTEMAS HÁPTICOS: ESTADO DEL ARTE}

Diversos prototipos e investigaciones se han desarrollado para la producción de percepciones táctiles por medio de actuadores, aplicados sobre la superficie de la yema de los dedos.

En el estudio presentado por Bau y colaboradores [12] se analiza la percepción de los diferentes estímulos de tipo vibratorio generados en una pantalla táctil transparente. La principal ventaja del método es la generación de vibraciones que conlleva a fricciones replicadas en el usuario, esto siempre y cuando la yema de los dedos se encuentre en movimiento. No obstante, el desarrollo planteado no proporciona diferentes estímulos para múltiples puntos de contacto, lo cual limita la resolución espacial a un área específica.

Por otro lado, Carter y colaboradores [13] presentan un sistema basado en transductores ultrasónicos, evidenciando una mejora en la percepción táctil, ya que no existe interacción física entre el actuador y la extremidad. Sin embargo, los autores concluyen que este tipo de dispositivos debe considerar y distinguir las separaciones entre estímulos para producir sensaciones en diversas regiones de la yema de los dedos.

En la investigación presentada por Lee y colaboradores [14], los autores implementan un actuador tipo láser 
produciendo sensaciones táctiles en zonas focales donde se ha dispuesto el actuador, además de ser un sistema compacto debido al tamaño del láser. No obstante, los autores concluyen que se debe generar un medio elástico apropiado para la transmisión de luz que sea indiferente a los cambios producidos en el aire, lo cual permitiría modificar en un cierto grado frecuencias y amplitudes para expandir el tipo de sensaciones.

Posteriormente en el trabajo de Kawasoe y colaboradores [15], los autores publican un artículo en donde se presenta la utilidad de un actuador piezo eléctrico, concluyendo que este tipo de transductores permiten dispositivos hápticos pequeños, que requieren un consumo de energía menor reflejado en parámetros de voltaje en el sistema, obteniéndose la reproducción de percepciones táctiles en regiones independientes de la yema de los dedos.

La utilización de estimulación eléctrica para procesos de excitación de las terminaciones nerviosas cutáneas a través de electrodos ha generado reportes enmarcados como hormigueos, vibraciones y estados de pulsaciones [16-17], a través de señales variantes en frecuencia, siendo utilizadas en diversas áreas de contacto del cuerpo humano [18-19].

En los trabajos de Kitamura y colaboradores [2021], se desarrolla la construcción de micro agujas para ser insertadas bajo la yema de los dedos. Este tipo de actuadores permite la generación de estímulos a un menor rango de voltaje debido a que se encuentran sobre la capa cornea y no en zona de contacto directa con la piel. De igual manera se reporta que la forma de las micro agujas afectará las percepciones del usuario relacionadas con la frecuencia e intensidad de la corriente aplicada.

Por su parte Yem y colaboradores [22], desarrollan un dispositivo háptico híbrido implementando un actuador mecánico tipo motor y un actuador eléctrico. Los autores concluyen que las percepciones producidas en el usuario se pueden enmarcar en presión y fricciones finas. De igual manera concluyen que combinando áreas de contacto $\mathrm{e}$ intensidad de corriente eléctrica se pueden obtener diversas sensaciones proyectadas hacia texturas de materiales debido a las deformaciones producidas en la yema de los dedos.
En el trabajo de Shima y colaboradores [23], se implementa la estimulación eléctrica para la reproducción de movimiento junto a un dispositivo tipo servo motor con el objetivo de reproducir en el usuario sensaciones de fuerza. Los autores concluyen que se deben considerar diversos puntos focales para ampliar la gama de percepciones debido a que el método propuesto se enfoca a la parte superior de la yema de los dedos.

Finalmente, en el trabajo de Geng y colaboradores [24], se acondiciona un método psicofísico para la evaluación de factibilidad de retroalimentación sensorial de umbrales por medio de estimulación eléctrica, clasificadas como detección, dolor y sensación notable. Los autores concluyen que este tipo de mecanismos puede ser implementado para sustituir la pérdida de sentido en usuarios amputados siempre que se controle frecuencias e intensidades en regiones de piel específicas.

\section{DISPOSITIVO DISEÑADO}

A continuación, se describe el dispositivo desarrollado para llevar a cabo el presente estudio.

\section{Hardware}

El sistema hardware (ver Figura 1), se compone de una placa de 12 centímetros de ancho por 10 centímetros de largo, la cual está conformada por tres etapas, constituidas por tres sub-etapas, descritas a continuación.

La primera sub-etapa consiste en un circuito generador de onda cuadrada por medio del dispositivo

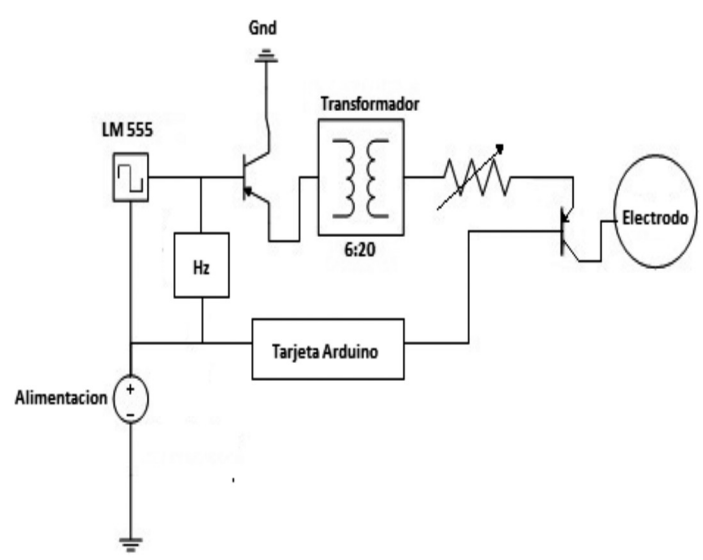

Figura 1. Diagrama general del hardware. 
LM555, en configuración monoestable, con frecuencias seleccionables a través de una tarjeta Arduino Mega 2560.

La segunda sub-etapa es un amplificador de voltaje obtenido de un transformador en configuración inversor.

Finalmente, la tercera sub-etapa está conformada por un arreglo de transistores que son conmutados de igual manera por la tarjeta Arduino para habilitar y deshabilitar los electrodos del actuador propuesto.

\section{Matriz de electrodos}

La matriz de electrodos construida consta de una distribución de 4 filas por 3 columnas, para un total de 12 pines, los cuales están dispuestos dentro de una cuadricula en una baquela transparente y flexible, apoyada sobre una espuma que brinda soporte al dispositivo, y permite ligeras deformaciones al aplicar presión (ver Figura 2).

Es de notar que cada pin electrodo tiene un diámetro de 3,0 milímetros, con una distancia entre centros de electrodos de 2,0 milímetros respectivamente; los nueve electrodos superiores son utilizados para estimulación, siendo declarados por las letras A, B, C, D, E, F, G, H, I.

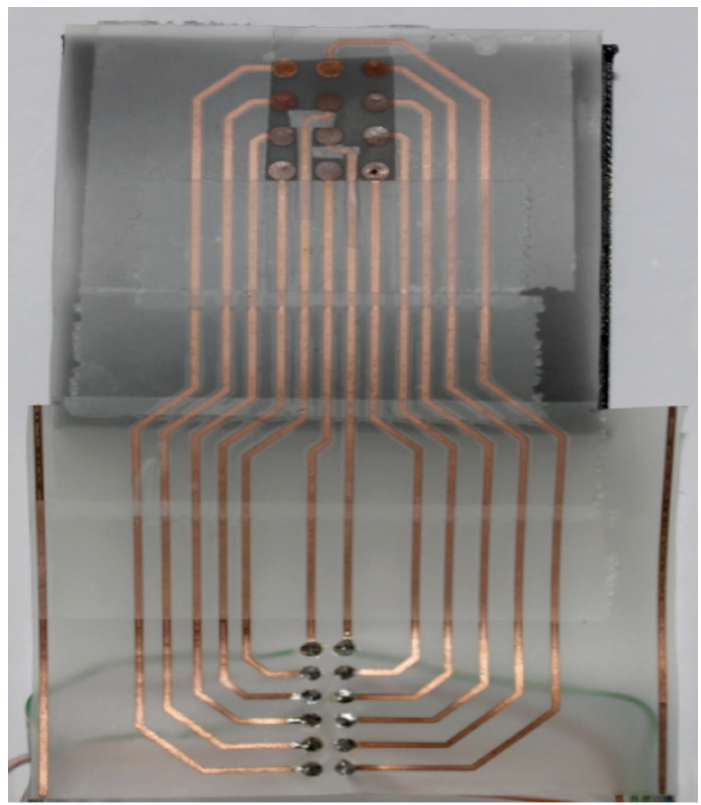

Figura 2. Matriz de electrodos 4 x3.
Los tres electrodos restantes permiten el direccionamiento de las señales provenientes de los transformadores. De igual manera esta matriz está desarrollada para que cubra una superficie de 1,5 centímetros por 2,5 centímetros, valores promedio de un dedo índice adulto (ver Figura 3).

\section{ENFOQUE EXPERIMENTAL}

El enfoque experimental es una adaptación realizada sobre las teorías básicas psicofísicas [25] el cual permite establecer y definir la tipología de las pruebas y los procedimientos previos a la aplicación de los experimentos planteados.

\section{Participantes}

El grupo de participantes está constituido por un total de 20 personas, las cuales se han separado en dos grupos de 10 personas cada uno, y con edades comprendidas entre 20 y 25 años. El protocolo establecido se divide en las siguientes etapas: adecuación, entrenamiento y ejecución.

\section{Adecuación}

La etapa de adecuación inicia con un proceso de limpieza por parte de cada uno de los usuarios,

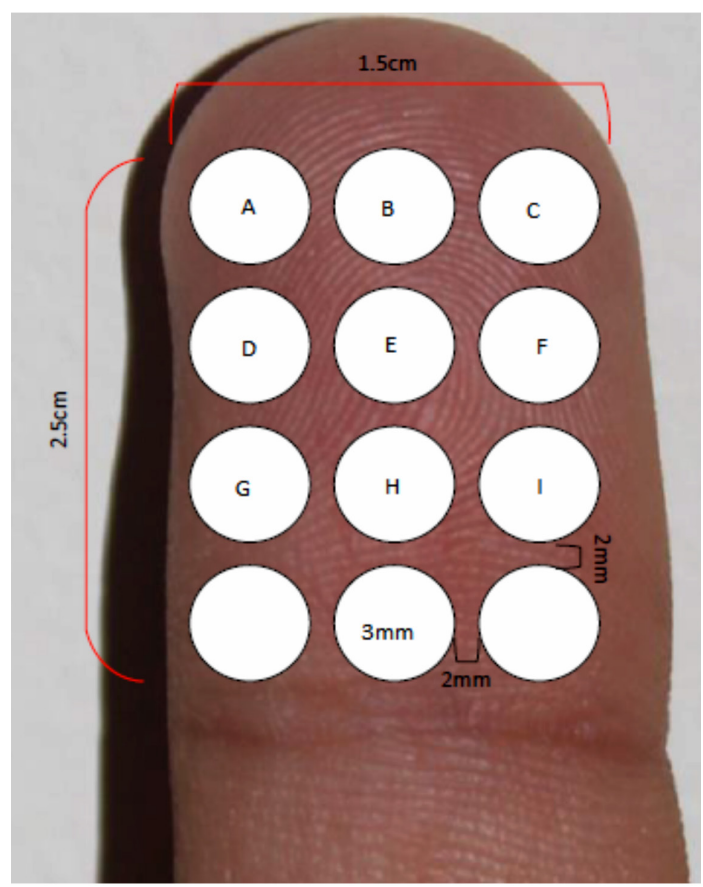

Figura 3. Dimensiones y disposiciones de electrodos. 
para ello deben lavarse las manos con agua y jabón, posteriormente deben frotarse la yema del dedo índice de la mano dominante por medio de un algodón que contiene alcohol etílico.

\section{Entrenamiento}

La etapa de entrenamiento se realiza con cada uno de los participantes, con el propósito de enseñarle a focalizar los estímulos, además de establecer psicológicamente un estado de costumbre frente a la estimulación eléctrica; este proceso de igual manera tiene como objetivo determinar la intensidad de la corriente promedio que es soportada por cada uno de ellos basados en percepciones o sensaciones que no produzcan dolor, o algún tipo de malestar en la yema de los dedos.

Este proceso se ejecuta realizando incrementos graduales de corriente sobre un electrodo. Una vez terminado el entrenamiento los usuarios expresaron que el valor de 20,78 microamperios era el adecuado debido a que no producía ningún tipo de molestias, además reportaron que era perceptible de forma constante en la extremidad.

\section{Ejecución}

La etapa de ejecución se encuentra conformada por 3 experimentos.

El primer experimento consta de tres secuencias fijas de estimulación, la primera secuencia se produce al habilitar el paso de señal eléctrica solo en el electrodo A, habilitando y deshabilitando el pin 3 veces. La segunda secuencia está dirigida hacia el electrodo D, y la tercera secuencia se ejecuta en el electrodo G; es de notar que este proceso se ha podido realizar con cada uno de los electrodos, no obstante, se seleccionaron estos tres pines para establecer una sensación vertical sin influencia de electrodos vecinos evaluando la percepción de estimulación en un solo punto a lo largo de la yema de los dedos.

El segundo experimento consta de dos secuencias de estimulación, la primera secuencia habilita el paso de señal eléctrica en el electrodo A, posteriormente en el electrodo B, y finalmente en el electrodo C. La segunda secuencia está dirigida hacia el electrodo D, a continuación, el electrodo E y luego al electrodo $\mathrm{F}$, es de notar que este proceso se ha podido realizar con diferentes combinaciones de electrodos, pero se seleccionaron estos pines para evaluar una sensación horizontal en la yema de los dedos.

El tercer experimento es una secuencia de estimulación fija, en donde se habilita el paso de señal en el electrodo G, luego se dirige hacia el electrodo I y finalmente la señal retorna al electrodo $G$, con el objetivo de evaluar la localización de estímulos en los extremos de la yema de los dedos, de igual manera este proceso ha podido realizarse a través de la matriz; esta secuencia se planteo debido a que los electrodos se encuentran rodeados de pines vecinos lo cual plantea una percepción detallada al momento de enunciar los puntos de focalización.

Para cada etapa de secuencias de estimulación se implementó un tiempo de 3 segundos, dejando un tiempo de descanso establecido en 5 veces el tiempo de excitación entre secuencias, basados en la literatura se han visto que autores tales como Konyo y colaboradores [26], Juárez y colaborador [27] especifican que los tiempos de estimulación no son estándar, así como los tiempos de descanso, por lo tanto se selecciona un tiempo de excitación inicial correspondiente a 3 segundos, con el fin de que el usuario capte los estímulos o sensaciones, de igual manera se incluye un tiempo de descanso determinado por 5 veces el tiempo de excitación con el objetivo de evitar calentamiento y deformaciones en la yema de los dedos, en este tiempo se debe reposar la yema de los dedos en una placa de vidrio, cada proceso se realizó a frecuencias de $25 \mathrm{~Hz}$, $50 \mathrm{~Hz}$ y $100 \mathrm{~Hz}$.

Además, los participantes deben utilizar auriculares para aumentar la concentración, aislándose del ruido externo. A continuación, se ilustra un usuario de cada grupo durante esta etapa; los componentes se describen a continuación, donde 1 corresponde a la fuente de alimentación, 2 a la matriz de electrodos, 3 la tarjeta Arduino, 4 el hardware implementado y 5 los auriculares (ver Figura 4).

\section{RESULTADOS}

La Figura 5 muestra los resultados del experimento 1. El promedio de aciertos frente a la zona de estimulación se ilustra sobre el eje vertical, y las frecuencias aplicadas sobre el eje horizontal. La relevancia estadística con respecto a los aciertos al aplicar diferentes frecuencias fue probada 


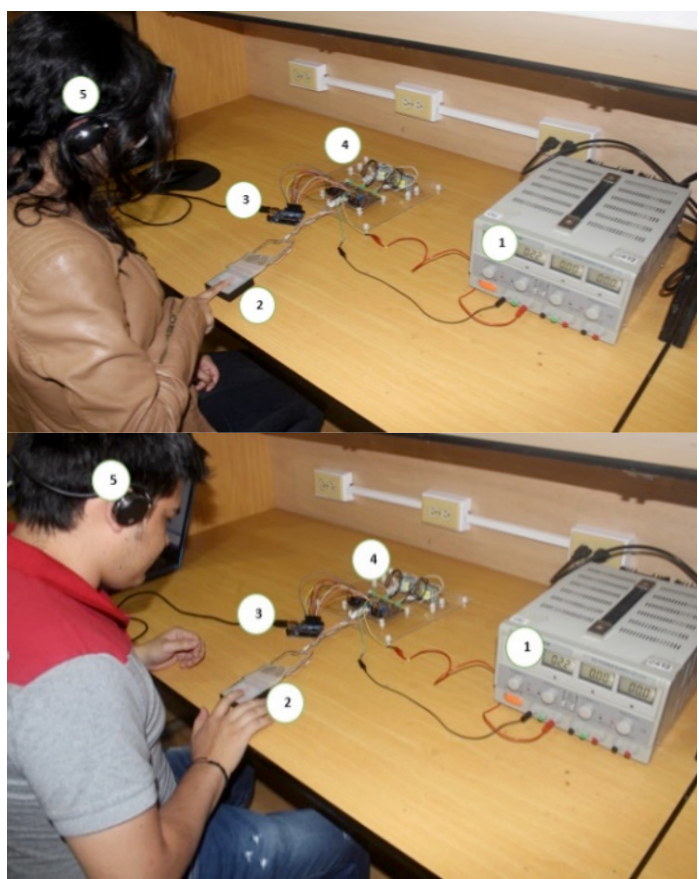

Figura 4. Participantes en etapa de ejecución.

mediante ANOVA [28], obteniéndose un valor de $p=0,52$ para las variaciones presentes respecto a los puntos, $p=0,001$ para las variaciones respecto a las frecuencias utilizadas y un $p=0,97$ con respecto a los grupos de participantes.

De los anteriores valores se puede concluir para este primer experimento que las activaciones de estimulación eléctrica a través de los pines A, D, G en la yema de los dedos se perciben sin dificultad.

De igual manera se llega a la conclusión que la aplicación de estímulos eléctricos en una zona específica a frecuencias bajas mantiene un promedio de aciertos alto, pero a medida que se aumentan estas frecuencias es más difícil percibir y localizar en que punto de la yema de los dedos se ejecuta la estimulación.

En la Figura 5 a) corresponde a estímulos en el punto $\mathrm{A}, \mathrm{b}$ ) corresponde a estímulos en el punto $\mathrm{D}$ y c) corresponde a estímulos en el punto G.

Por su parte la Figura 6 muestra los resultados del experimento 2 .

Con los datos se obtuvo un $p=0,13$ para las variaciones respecto a los pines, un $p=0,004$ para las variaciones respecto a las frecuencias seleccionas y un $p=0,73$ con respecto a los grupos de participantes.

De lo anterior se puede concluir que las secuencias planteadas para generar sensaciones de forma horizontal aplicadas en la yema de los dedos se perciben sin dificultad, pero si se ven afectados por las frecuencias seleccionadas.

A partir del análisis se llega a la conclusión que al aplicarse una secuencia de estímulos con un sentido desde izquierda hacia derecha el promedio de aciertos tiende a disminuir a medida que se aumenta las frecuencias; tanto la primera secuencia establecida por los pines A, B,C como la secuencia D,E,F, presenta el mayor numero de aciertos a los $25 \mathrm{~Hz}$, es de notar que los usuarios manifestaron que este experimento recrea la sensación táctil de desplazamiento por medio de la yema de los dedos con un objeto, manifestaron de igual forma que en la frecuencia de $100 \mathrm{~Hz}$ se recrea una sensación cuando se manipula un material liso, aspectos a considerarse en los trabajos futuros. a)

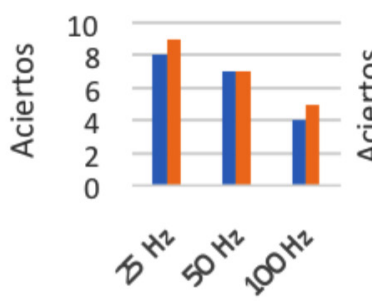

b)

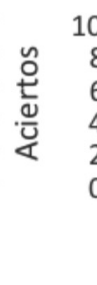

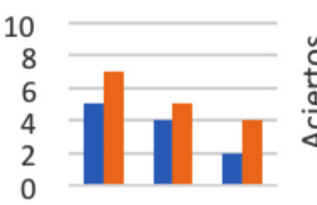

2550100 $\mathrm{Hz} \quad \mathrm{Hz} \quad \mathrm{Hz}$ c)

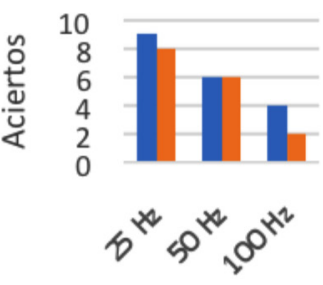

\section{Frecuencias}

- Hombres Mujeres

Figura 5. Resultados experimento 1. 
En la Figura 6 a) corresponde a estímulos en los puntos A, B, C, b) corresponde a estímulos en los puntos D, E, F.

Finalmente, para el último experimento, la Figura 7 muestra los resultados, obteniéndose un $p=0,18$ respecto a los grupos de participantes, y un valor de $p=0,01$ para las variaciones respecto a las frecuencias seleccionadas.

Se llega a la conclusión de que al aplicarse una secuencia de estímulos en los electrodos inferiores que abarcan la yema de los dedos, las respuestas de aciertos son mayores en la frecuencia de $25 \mathrm{~Hz}$ registrando una leve disminución a los $50 \mathrm{~Hz}$, de igual manera se presenta calificaciones de aciertos en la frecuencia de $100 \mathrm{~Hz}$.

La Figura 7 a) corresponde a estímulos en los puntos $\mathrm{G}, \mathrm{I}, \mathrm{G}$ de la secuencia utilizada.

\section{CONCLUSIONES Y TRABAJOS FUTUROS}

En este estudio se ha desarrollado un procedimiento de evaluación que combina el sentido del tacto con

a)

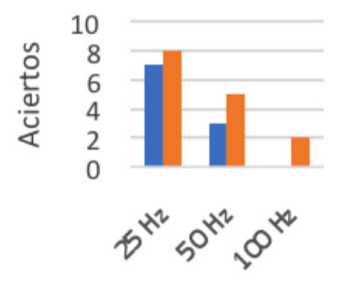

b)

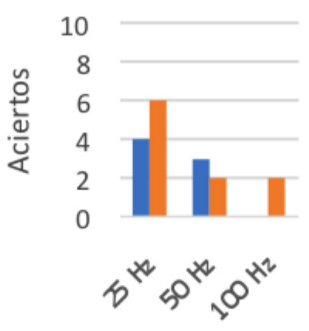

Frecuencias

- Hombres Mujeres

Figura 6. Resultados experimento 2.

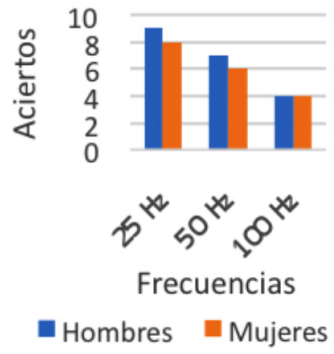

Figura 7. Resultados experimento 3. interpretaciones psicofísicas, para la percepción de estímulos en diversos puntos focales de la yema de los dedos.

La distribución de electrodos realizada sobre la matriz flexible y transparente permite abarcar la superficie de la yema de los dedos evaluando comportamientos de localización a través de puntos fijos y combinaciones.

La experimentación establece que las secuencias planteadas fueron percibidas sin dificultades en los participantes, donde las frecuencias se convierten en un factor influyente en los aciertos de localización de cada pin, y a medida que este factor aumentaba dichos aciertos tendían a disminuir.

Como trabajos futuros se prevé la comparación de las distancias entre electrodos para la evaluación de la percepción con diferentes matrices. Además, se plantea considerar otros factores dentro de las evaluaciones de localización táctil, tales como intensidad de corriente variable, tiempos de estimulación y edad de los participantes.

De igual manera se plantea que la estimulación eléctrica puede recrear sensaciones de texturas presentes en diversos materiales, debido a lo manifestado por los participantes en el experimento 2 , aplicando un reconocimiento activo por parte de la yema de los dedos.

\section{REFERENCIAS}

[1] W. Barfield. "The use of haptic display technology in education". Themes in Science and Technology Education. Vol. 2, pp. 11-30. 2009.

[2] M. Eid, M. Orozco and A. El Saddik. "A guided tour in haptic audio-visual environments and applications". International Journal of Advanced Media and Communication. Vol. 1, pp. 265-297. 2007.

[3] P. Do and D. Homa. "Haptics rendering and applications". Haptics Rendering and Applications. 2012.

[4] M. Mengoni, B. Colaiocco, M. Germani and M. Peruzzini. "Design of a novel human-computer interface to support HCD application". ASME 2010 International Design Engineering Technical Conferences and 
Computers and Information in Engineering Conference, pp. 15-17. 2010.

[5] V. Chouvardas, A. Miliou and M. Hatalis. "Tactile displays: overview and recent advances". Displays. Vol. 29, pp. 185-194. 2008.

[6] R. Johansson and A. Vallbo. "Tactile sensibility in the human hand: relative and absolute densities of four types of mechanoreceptive units in glabrous skin". The Journal of Physiology, pp. 283-300. 1979.

[7] D. Pamungkas and K. Ward. "Tele operation of a robot arm with electro tactile feedback". IEEE International Conference on Advanced Intelligent Mechatronics, pp. 704-709. 2013.

[8] K. Kaczmarek, J. Webster and R. Radwin. "Maximal dynamic range electrotactile stimulation waveforms". IEEE Transactions on Biomedical Engineering. Vol. 39, pp. 701715. 1992.

[9] H. Culbertson, B. Schorr and A. Okamura. "Haptics: the present and future of artificial touch sensation". Annual Review of Control, Robotics, and Autonomous Systems. 2018.

[10] K. Kaczmarek, J. Webster, P. Bach and W. Tompkins. "Electrotactile and vibrotactile displays for sensory substitution systems". IEEE Transactions on Biomedical Engineering. Vol. 38, pp. 1-16. 1991.

[11] A. Szeto and R. Riso. "Sensory feedback using electrical stimulation of the tactile sense". Rehabilitation Engineering, pp. 29-78. 1990.

[12] O. Bau, I. Poupyrev and C. Harrison. "TeslaTouch: electrovibration for touch surfaces". Proceedings of the 23nd Annual ACM Symposium on User Interface Software and Technology, pp. 283-292. 2010.

[13] T. Carter, S. Seah, B. Long, B. Drinkwater and S. Subramanian. "Ultrahaptics: multi point mid air haptic feedback for touch surfaces". Proceedings of the 26nd Annual ACM Symposium on User Interface Software and Technology, pp. 505-514. 2013.

[14] H. Lee, J. Kim, S.Choi, J. Hoon, J. Park, A. Kim, H. Oh and S. Chung. "Mid air tactile stimulation using laser induced thermoelastic effects: The first study for indirect radiation". IEEE World Haptics Conference, pp. 374380. 2015.

[15] M. Kawazoe, Y. Kosemura and N. Miki. "Encoding and presentation of surface textures using mechanotactile display". Sensors Actuators. Vol. 261, pp. 30-39. 2017.

[16] K. Kaczmarek and P. Bach. "Tactile displays". Virtual Environments and Advanced Interface Design, pp. 349-414. 1995.

[17] J. Slopsema, J. Boss, L. Heyboer, C. Tobias, B. Draggoo and K. Polasek. "Natural sensations evoked in distal extremities using surface electrical stimulation". The Open Biomedical Engineering Journal, pp. 1-15. 2018.

[18] H. Kajimoto, N. Kawakami, S. Tachi and M. Inami. "SmartTouch: electric skin to touch the untouchable". IEEE Computer Graphics and Applications, pp. 36-43. 2004.

[19] A. Yamamoto, S. Nagasawa and T. Higuchi. "Electrostatic tactile display with thin film slider and its application to tactile telepresentation systems". IEEE Transactions on Visualization and Computer Graphics. Vol. 12, pp. 168-177. 2006.

[20] N. Kitamura, J. Chim and N. Miki. "Micro needle electrode array for electro tactile display". Solid State Sensors, Actuators and Microsystems, pp. 106-107. 2013.

[21] N. Kitamura, J. Chim and N. Miki”. Electrotactile display using microfabricated micro-needle array". Journal of Micromechanics and Microengineering. Vol. 25. 2015.

[22] V. Yem and H. Kajimoto. "Wearable tactile device using mechanical and electrical stimulation for fingertip interaction with virtual world". IEEE Virtual Reality, pp. 99104. 2017.

[23] K. Shima and R. Sato. "A novel haptic device design based on somatosensory superimposed stimuli". Advanced Robotics. Vol. 31, pp. 135-142. 2017.

[24] B. Geng, J. Dong, W. Jensen, S. Dosen, D. Farina and N. Kamavuako. "Psychophysical evaluation of subdermal electrical stimulation in relation to prosthesis sensory feedback". IEEE Transactions on Neural Systems and Rehabilitation Engineering. Vol. 26, pp. 709715. 2018.

[25] W. Ehrenstein and A. Ehrenstein. "Psychophysical methods". Modern Techniques in Neuroscience Research, pp. 1211-1241. 1996.

[26] M. Konyo, S. Tadokoro, A. Yoshida and N. Saiwaki. "A Tactile Synthesis Method Using 
Multiple Frequency Vibrations for Representing Virtual Touch". International Conference on Intelligent Robots and Systems. 2005.

[27] E. Juárez y O. Oliva. "Diseño de un estimulador muscular para terapias analgésicas de baja frecuencia”. Escuela superior de ingeniería mecánica y eléctrica. Instituto politécnico nacional. México. 2012.
[28] H. Keselman, C. Huberty, L. Liz, S. Olejnik, R. Cribbie, B. Donahue, R. Kowalchuk, L. Lowman, M. Petoskey, J. Keselman and J. Levin. "Statistical practices of educational researchers: An analysis of their anova, mancova, and ancova analyses". American Educational Research Association. Vol. 68, pp. 350-386. 1998. 